\title{
Convex bodies in Euclidean and Weil-Petersson geometries
}

\author{
Sumio Yamada
}

Supported in part by JSPS Grant-in-aid for Scientific Research No.20540201

\section{Introduction}

Given an open convex body $\Omega$ in a Euclidean space, Hilbert in 1895 (8]) proposed a natural metric $H(x, y)$, now called Hilbert metric, defined on $\Omega$, as the logarithm of the cross ratio of a quadruple, $x, y, b(x, y)$ and $b(y, x)$, where $b(x, y)$ is where the ray from $x$ through $y$ hits the boundary of $\Omega$.

For historical reasons, and in order to be consistent with the existing literature, we use the term "metric" in place of distance function; it does not refer to a Riemannian metric. To be more precise, in this article we define a metric on a set $X$ to be a function $\delta: X \times X \rightarrow\left(\mathbf{R}_{+} \cup\{\infty\}\right.$ satisfying:

1. $\delta(x, x)=0$ for all $x$ in $X$,

2. $\delta(x, z) \leq \delta(x, y)+\delta(y, z)$ for all $x, y$ and $z$ in $X$.

In [14], for example, a function satisfiying the above is named weak metric. Note in this definition neither the symmetry $\delta(x, y)=\delta(y, x)$ nor the nondegeneracy $\delta(x, y)=0 \Rightarrow x=y$ is assumed.

Then the logarithm of the cross ratio as above indeed defines a metric, which is Finslerian and projective. A Finsler structure on a Euclidean space determines a norm to each tangent space, and the norm itself is called Minkowski functional. A metric is said to be projective when Euclidean straight lines are geodesic. The unit disc with its Hilbert metric $H(x, y)$ is a prominent example; Klein's model for the hyperbolic plane.

As the value $H(x, y)$ can be written as

$$
\log \frac{|x-b(x, y)||y-b(y, x)|}{|y-b(x, y)||x-b(y, x)|}=\log \frac{|x-b(x, y)|}{|y-b(x, y)|}+\log \frac{|y-b(y, x)|}{|x-b(y, x)|}
$$

Funk [7] looked at the first term of the right hand side above as a metric, even though it is asymmetric, which has been called Funk metric. The reader is referred to [14, 15, where the historical and technical backgrounds are presented comprehensively. 
The purpose of this article is threefold. The first is to introduce a new variational characterization (Theorem 1) of the Funk metric for a convex domain $\Omega \subset \mathbf{R}^{d}$. It provides a new and short proof of the triangle inequality for the Funk and Hilbert distances. While the original proof by Hilbert uses the projective invariance of the cross ratio, the new one is less dependent on the Euclidean geometry, more on the convexity of the distance function. The new definition of the Funk metric also leads to new proofs for a set of results previously obtained in [15] concerning the geometry of convex curves $\partial \Omega$, as well as a new convexity statement of the functional $F\left(x_{0}, x\right)$ in the $x$ variable. Moreover we demostrate three different ways of writing down the Funk metric (and consequently the Hilbert metric) in this article.

Secondly we generalize the Funk and Hilbert metrics to Teichmüller spaces of closed surfaces of genus $g>1$, where the background geometry is the one induced by the Weil-Petersson metric (see, for example, 20]) instead of the Euclidean geometry. The Weil-Petersson geometry of Teichmüller spaces has been known to possess many convex functionals (17, 21]) in addition to the Weil-Petersson distance function itself. This feature has led to a construction of a new space (23]), called Teichmüller-Coxeter complex, within which the original Teichmüller space sits as an open convex subset. This setting naturally allows us to adapt the Euclidean setting for the Funk metric (Theorem 2) as well as the Hilbert metric to the Weil-Petersson setting. In doing so, we introduce three different (weak) metrics $F_{1}, F_{2}$ and $F_{3}$, each corresponding to one of the three representations of the Funk metric in the Euclidean setting, and each distinct from each other. The differences are caused by the negative sectional curvature of the Weil-Petersson metric tensor. The Weil-Petersson Funk metrics (as well as the Weil-Petersson Hilbert metrics) thus defined are all invariant under the mapping class group action.

The last aim is to draw the reader's attention to a similarity between the newly defined Weil-Petersson Funk metric $F_{2}$ and so-called Thurston's asymmetric metric. In his paper [16, Thurston embeds the Teichmüller space to its cotangent space as a convex body, whose boundary is identified with the set of projective measured laminations on the given surfaces. There an asymmetric metric is introduced, by taking the logarithm of the ratio of hyperbolic lengths of a simple closed geodesic. In the last section of this article, we will demonstrate a similarity between the Weil-Petersson Funk metric and Thurston's metric, which would lead to some questions and speculations. When the closed surface is a torus $(g=1)$, Thurston's metric has been analyzed closely in 4 and it is effectively compared to the geometry of the Teichmüller metric. We recall that on the Teichmüller space for the torus, the Teichmüller metric and the Weil-Petersson metric are isometric.

The author would like to thank Athanase Papadopoulos for many helpful comments, and the referee for the careful reading and the criticisms. 


\section{Euclidean Geometry}

\subsection{Funk metric}

Suppose that $\Omega$ is an open convex subset in a Euclidean space $\mathbf{R}^{d}$. In what follows, we set the presentation by Papadopoulos and Troyanov [14] as our reference for Funk and Hilbert metrics.

First we represent the convex set $\Omega$ as $\cap_{\pi(b) \in \mathcal{P}} H_{\pi(b)}$ where $H_{\pi(b)}$ is the half space bounded by a supporting hyperplane $\pi(b)$ of $\Omega$ at the boundary point $b$, containing the convex set $\Omega$. The index set $\mathcal{P}$ is the set of all supporting hyperplanes of $\Omega$. That for every boundary point $p$ there exists a supporting hyperplane $\pi(b)$ follows from the definition of convexity of $\Omega$. In general, there can be more than one supporting hyperplane of $\Omega$ at $p \in \partial \Omega$. The index set $\mathcal{P}$ is identified with the set of unit normal vectors to the supporting hyperplanes. It is identified with a subset of $S^{d-1}$, is equal to the entire sphere when the convex set is bounded. We denote by $\mathcal{P}(b)$ the set of supporting hyperplanes at $b \in \partial \Omega$.

Definition For a pair of points $x$ and $y$ in $\Omega$, the Funk asymmetric metric [7] is defined by

$$
F(x, y)=\log \frac{d(x, b(x, y))}{d(y, b(x, y))} .
$$

where the point $b(x, y)$ is the intersection of the boundary $\partial \Omega$ and the ray $\left\{x+t \xi_{x y} \mid t>0\right\}$ from $x$ though $y$. Here $\xi_{x y}$ is the unit vector along the ray.

Now let $\pi_{0}$ be a supporting hyperplane at $b(x, y)$, namely $\pi_{0} \in \mathcal{P}(b(x, y))$. Then note the similarity of the triangle $\triangle\left(x, \Pi_{\pi_{0}}(x), b(x, y)\right)$ and $\triangle\left(y, \Pi_{\pi_{0}}(y), b(x, y)\right)$, where $\Pi_{\pi_{0}}(p)$ is the foot of the point $p$ on the hyperplane $\pi_{0}$, or put it differently $\Pi_{\pi_{0}}: \mathbf{R}^{d} \rightarrow \pi_{0}$ is the nearest point projection map. This says that

$$
\log \frac{d(x, b(x, y))}{d(y, b(x, y))}=\log \frac{d\left(x, \pi_{0}\right)}{d\left(y, \pi_{0}\right)} .
$$

Also by the similarity argument of triangles, note that the right hand side of the equality is independent of the choice of $\pi_{0}$ in $\mathcal{P}(b(x, y))$.

Using the convexity of $\Omega$, the quantity $F(x, y)$ can be characterized variationally as follows. Define $T(x, \xi, \pi)$ by $\pi \cap\{x+t \xi \mid t>0\}$ with $\pi \in \mathcal{P}$. Consider the case $\xi=\xi_{x y}$. When the hyperplane supports $\Omega$ at $p$, we have $T\left(x, \xi_{x y}, \pi\right)=$ $b(x, y)$ and otherwise the point $T\left(x, \xi_{x y}, \pi\right)$ lies outside $\Omega$. When $\pi \notin \mathcal{P}(b(x, y))$, by the similarity argument between the triangles $\triangle\left(x, F_{\pi}(x), T\left(x, \xi_{x y}, \pi\right)\right)$ and $\triangle\left(y, F_{\pi}(y), T\left(\xi_{x y}, \pi\right)\right)$ again we have

$$
\frac{d(x, \pi)}{d(y, \pi)}=\frac{d\left(x, T\left(x, \xi_{x y}, \pi\right)\right)}{d\left(y, T\left(x, \xi_{x y}, \pi\right)\right)} .
$$

Note that the closest point to $x$ along the ray $\left\{x+t \xi_{x y}: t>0\right\}$ of the form $T\left(x, \xi_{x y}, \pi\right)$ is $b(x, y)$. This in turn says that a hyperplane $\pi$ which supports $\Omega$ at $b(x, y)$ maximizes the ratio $d\left(x, T\left(x, \xi_{x y}, \pi\right)\right) / d\left(y, T\left(x, \xi_{x y}, \pi\right)\right)$ among all the elements of $\mathcal{P}$; 


$$
\log \frac{d(x, b(x, y))}{d(y, b(x, y))}=\sup _{\pi \in \mathcal{P}} \log \frac{d(x, \pi)}{d(y, \pi)} .
$$

Hence we have a new characterization of the Funk metric;

Theorem 1 The Funk metric defined as above over a convex subset $\Omega \subset \mathbf{R}^{d}$ has the following variational formulation;

$$
F(x, y)=\sup _{\pi \in \mathcal{P}} \log \frac{d(x, \pi)}{d(y, \pi)} .
$$

With this formulation, one can readily see that $F(x, y)$ satisfies the triangle inequality, for

$$
\begin{gathered}
F(x, y)+F(y, z)=\sup _{\pi \in \mathcal{P}} \log \frac{d(x, \pi)}{d(y, \pi)}+\sup _{\pi \in \mathcal{P}} \log \frac{d(y, \pi)}{d(z, \pi)} \\
\geq \sup _{\pi \in \mathcal{P}}\left(\log \frac{d(x, \pi)}{d(y, \pi)}+\log \frac{d(y, \pi)}{d(z, \pi)}\right)=\sup _{\pi \in \mathcal{P}} \log \frac{d(x, \pi)}{d(z, \pi)}=F(x, z)
\end{gathered}
$$

Note that the triangle inequality becomes an equality when

$$
\sup _{\pi \in \mathcal{P}} \log \frac{d(x, \pi)}{d(y, \pi)}+\sup _{\pi \in \mathcal{P}} \log \frac{d(y, \pi)}{d(z, \pi)}=\sup _{\pi \in \mathcal{P}}\left(\log \frac{d(x, \pi)}{d(y, \pi)}+\log \frac{d(y, \pi)}{d(z, \pi)}\right)
$$

is satisfied. For this to occur, we only need $\mathcal{P}(b(x, y)) \cap \mathcal{P}(b(y, z)) \neq \emptyset$. Let $\pi_{0}$ be an element in the set $\mathcal{P}(b(x, y)) \cap \mathcal{P}(b(y, z)) \neq \emptyset$. Then the boundary point $b(x, y)$ and $b(y, z)$ share the same supporting hyperplane $\pi_{0}$, and we have

$$
\sup _{\pi \in \mathcal{P}} \log \frac{d(x, \pi)}{d(y, \pi)}=\log \frac{d\left(x, \pi_{0}\right)}{d\left(y, \pi_{0}\right)}, \quad \sup _{\pi \in \mathcal{P}} \log \frac{d(y, \pi)}{d(z, \pi)}=\log \frac{d\left(y, \pi_{0}\right)}{d\left(z, \pi_{0}\right)}
$$

and

$$
\sup _{\pi \in \mathcal{P}} \log \frac{d(x, \pi)}{d(z, \pi)}=\log \frac{d\left(x, \pi_{0}\right)}{d\left(z, \pi_{0}\right)} .
$$

inducing the equality. The observation is equivalent to the following statement;

Proposition 8.3 [14] Let $\Omega$ be an open convex subset of $\mathbf{R}^{d}$ such that $\partial \Omega$ contains some non degenerate Euclidean segment $[p, q]$ and let $x$ and $z$ be two distinct points in $\Omega$ such that $\left\{t \xi_{x z} \mid t \geq 0\right\} \cap[p, q] \neq \emptyset$. Let $\Omega^{\prime}$ be the intersection of $\Omega$ with the affine subspace of $\mathbf{R}^{d}$ spanned by $\{x\} \cup[p, q]$. Then, for any point $y$ in $\Omega^{\prime}$ satisfying $\left\{t \xi_{x y} \mid t \geq 0\right\} \cap[p, q] \neq \emptyset$ and $\left\{t \xi_{y z} \mid t \geq 0\right\} \cap[p, q] \neq \emptyset$, we have $F(x, y)+F(y, z)=F(x, z)$.

A notable situation when one has $\mathcal{P}(b(x, y)) \cap \mathcal{P}(b(y, z)) \neq \emptyset$ is when $x, y$ and $z$ are colinear, with $y$ lying between $x$ and $z$. This in turn says that the straight lines are Funk geodesics, or in Hilbert's term, the Funk metric is projective. This corresponds to Corollary 8.2 of [14]. Here a path $s:[a, b] \rightarrow$ 
$(X, d)$ in a metric space $(X, d)$ is said to be geodesic when for any $a<t<b$, $F(s(a), s(t))+F(s(t), s(b))=F(s(a), s(b))$ is satisfied.

On the other hand, when $\pi_{0}$ is in the set $\mathcal{P}(b(x, y)) \cap \mathcal{P}(b(y, z))$ the concatenation of the line segment $\overline{x y}$ and $\overline{y z}$ is also a Funk geodesic, a situation occurring when the boundary set $\partial \Omega$ has a straight edge; a statement corresponding to Corollary 8.4 of [14.

We next consider the complementary situation where $\mathcal{P}\left(b_{1}\right) \cap \mathcal{P}\left(b_{2}\right)=\emptyset$ for a pair of distinct points $b_{i} \in \partial \Omega$. Geometrically this characterizes strict convexity of the domain $\Omega$, namely the boundary $\partial \Omega$ contains no closed line segments. From the preceding argument, it follows that the only way the equality for the triangle inequality occurs is when the three points $x, y$ and $z$ are co-linear. Hence for strictly convex domains, the Funk geodesics consists of line segments only, or equivalently, given a pair of points there is a unique Funk geodesic. This corresponds to Corollary 8.8 of [14].

With the additional assumption that the domain is strictly convex, the following convexity of the Funk distance can be formulated. Namely consider a geodesic/line $s(t)$ in $\Omega \subset \mathbf{R}^{d}$. Then the function $F(x, s(t))$ is convex in the parameter $t$; first fix a supporting hyperplane $\pi$ in $\mathcal{P}$. Then it follows that

$$
\frac{d}{d t} \log \frac{d(x, \pi)}{d(s(t), \pi)}=-\frac{\left\langle-\nu_{\pi}(s(t)), \dot{s}(t)\right\rangle}{d(s(t), \pi)}, \frac{d^{2}}{d t^{2}} \log \frac{d(x, \pi)}{d(s(t), \pi)}=\frac{\left\langle-\nu_{\pi}(s(t)), \dot{s}(t)\right\rangle^{2}}{d(s(t), \pi)^{2}}
$$

Here $\nu_{\pi}(x)$ is the unit vector at $x$ perpendicular to the hypersurface $\pi(b)$ oriented toward $\pi$. In particular $-\nu_{b}(x)$ is the gradient vector of the function $d(x, \pi)$

So $\log \frac{d(x, \pi)}{d(s(t), \pi)}$ is convex in $t$ for each $\pi \in \mathcal{P}$. By taking supremum over $\pi \in$ $\mathcal{P}$, the resulting function $F(x, s(t))$ is convex in $t$. We note that the convexity of the geodesic ball $\left\{y \in \Omega \mid F_{\Omega}(x, y)<\delta\right\}$ centered at $x$ has been known (Proposition 8.11 (1) of [14 ), which is equivalent to the convexity of $F(x, s(t)$ ) in $t$ for any lines $s(t)$ with $s(0)=x$.

Next consider the function

$$
F(s(t), x)=\sup _{\pi \in \mathcal{P}} \log \frac{d(s(t), \pi)}{d(x, \pi)}
$$

in $t$. The first and second derivatives of this function are given by

$$
\frac{d}{d t} \log \frac{d(s(t), \pi)}{d(x, \pi)}=\frac{\left\langle-\nu_{\sigma}(s(t)), \dot{s}(t)\right\rangle}{d(s(t), \pi)}, \frac{d^{2}}{d t^{2}} \log \frac{d(s(t), \pi)}{d(x, \pi)}=-\frac{\left\langle-\nu_{\sigma}(s(t)), \dot{s}(t)\right\rangle^{2}}{d(s(t), \pi)^{2}}
$$

This says that the Funk distance $F(s(t), x)$ is not convex in $t$.

\section{$2.2 \quad$ Finsler structure}

We next consider the linear structure of the Funk metric, by identifying it with a Finsler norm. The tautological weak Finsler structure [14] is given by the 
following Minkowski functional $p_{\Omega, x}(\xi)=\frac{1}{r_{\Omega, x}(\xi)}$ with the radial function of $\Omega$ defined by

$$
r_{\Omega, x}(\xi)=\sup \{t \in \mathbf{R} \mid x+t \xi \in \Omega\} .
$$

From our exposition so far, we can identify the value of $r_{\Omega, x}(\xi)$ as

$$
r_{\Omega, x}(\xi)=\inf _{\pi \in \mathcal{P}} d(x, T(x, \xi, \pi))=\inf _{\pi \in \mathcal{P}} \frac{d(x, \pi)}{\left\langle\nu_{\pi}(x), \xi\right\rangle} .
$$

Note that the functional

$$
p_{\Omega, x}(\xi)=\sup _{\pi \in \mathcal{P}} \frac{\left\langle\nu_{\pi}(x), \xi\right\rangle}{d(x, \pi)}
$$

is convex in $\xi \in T_{x} \mathbf{R}^{d}$, as the functional $\frac{\left\langle\nu_{\pi}(x), \xi\right\rangle}{d(x, \pi)}$ is convex (linear in particular) in $\xi$ for each fixed $\pi \in \mathcal{P}$, and by taking sup over $\pi$, the convexity is preserved. In Theorem 6.1 of [14, the infimum, among all the piecewise $C^{1}$ paths with given end points, of the length according to the Finsler norm is shown to coincide with the Funk metric. The proof follows from the fact that for a fixed $\pi$, the function $\frac{\left\langle\nu_{\pi}(\sigma(t)), \dot{\sigma}(t)\right\rangle}{d(\sigma(t), \pi)}$ is the $t$-derivative of $\log \frac{d(x, \pi)}{d(\sigma(t), \pi)}$ for a $C^{1}$-path $\sigma(t)$.

\section{$2.3 \quad$ A Brief Summary}

We have introduced three different representations of the Funk metric. The first one is the original definition by Funk [7];

$$
F_{1}(x, y)=\log \frac{d(x, b(x, y))}{d(y, b(x, y))}
$$

where the point $b(x, y)$ is the intersection of the boundary $\partial \Omega$ and the ray $\left\{x+t \xi_{x y}: t>0\right\}$ from $x$ though $y$.

The second one is the variational interpretation of the value above using the geometry of supporting hyperplanes;

$$
F_{2}=\sup _{\pi \in \mathcal{P}} \log \frac{d(x, \pi)}{d(y, \pi)}
$$

where $\mathcal{P}$ is the set of all supporting hyperplanes of $\Omega$.

And lastly the Finsler structure $p_{\Omega, x}(\xi)$ is identified so that the Funk distance is described as the infimum of length of curves;

$$
F_{3}=\inf _{\sigma} \int_{a}^{b} p_{\Omega, \sigma(t)}(\dot{\sigma}(t)) d t
$$

where the infimum is taken over all the piecewise $C^{1}$ curves with $\sigma(a)=x$ and $\sigma(b)=y$.

We emphasize that they are all equal to each other

$$
F(x, y):=F_{1}(x, y)=F_{2}(x, y)=F_{3}(x, y)
$$

for a convex domain $\Omega \subset \mathbf{R}^{d}$. 


\subsection{Hilbert metric}

We symmetrize the Funk metric by taking the arithmetic means

$$
H(x, y)=\frac{1}{2}(F(x, y)+F(y, x))
$$

This metric is called Hilbert metric on $\Omega$.

For a convex set $\Omega$ in $\mathbf{R}^{d}$, the affine line segment connecting $x$ and $y$ is a Funk geodesic realizing both lengths $F(x, y)$ and $F(y, x)$. This says that the line segment also is the Hilbert geodesic.

The Hilbert metric satisfies the triangle inequality which is obtained by taking the sum of the following pair of inequalities;

$$
F(x, y)+F(y, z) \geq F(x, z)
$$

which has been shown above and

$$
F(y, x)+F(z, y) \geq F(z, x)
$$

which follows from the similar argument;

$$
\begin{gathered}
F(y, x)+F(z, y)=\sup _{\pi \in \mathcal{P}} \log \frac{d(y, \pi)}{d(x, \pi)}+\sup _{\pi \in \mathcal{P}} \log \frac{d(z, \pi)}{d(y, \pi)} \\
\geq \sup _{\pi \in \mathcal{P}}\left(\log \frac{d(y, \pi)}{d(x, \pi)}+\log \frac{d(z, \pi)}{d(y, \pi)}\right)=\sup _{\pi \in \mathcal{P}} \log \frac{d(z, \pi)}{d(x, \pi)}=F(z, x) .
\end{gathered}
$$

Note this proof differs from Hilbert's which uses the projective property of the cross ratio among the points $x, y, b(x, y)$ and $b(y, x)$.

Recall that for a strictly convex $\Omega, F(x, p(t))$ is convex in $t$ and the convexity of $F(p(t), x)$ is inconclusive in general. As for the Hilbert metric where $F(x, p(t))$ and $F(p(t), x)$ are averaged, there is a complete characterization of $\Omega$ for which it is convex. Suppose now that the Hilbert distance $H(x, p(t))$ of a given $\Omega$ is convex in $t$. Then it is equivalent to saying that the geometry is nonpositively curved in the sense of Busemann, namely given a point $p$, there exists a neighborhood $U$ of $p$ such that for any pair $x$ and $y, 2 H\left(m_{x}, m_{y}\right) \leq H(x, y)$ where $m_{x}$ is the midpoint with respect to $H$ between $x$ and $p$. Under this hypothesis P.Kelly and E.G.Strauss [10] showed that $\Omega$ is an ellipsoid, which is projectively equivalent to the unit disc. Hence the metric space $(\Omega, H)$ is isometric to the Poincaré disk.

\section{Weil-Petersson Geometry}

\section{$3.1 \quad \overline{\mathcal{T}}$ as a convex subset in $D(\overline{\mathcal{T}}, \iota)$}

Now, in the Weil-Petersson geometric setting, first recall that given a closed surface $\Sigma$ of genus $g>1$, the Weil-Petersson metric completion( $(22]) \overline{\mathcal{T}}$ is identified with the augmented Teichmüller space [1, 11,

$$
\overline{\mathcal{T}}=\cup_{\sigma \in \mathcal{C}(\Sigma)} \mathcal{T}_{\sigma}
$$


where $\mathcal{C}(\Sigma)$ is the curve complex of $\Sigma$, which can be identified as the index set of nodal surfaces $\Sigma_{\sigma}$ 's specifying the location of the nodes. $\mathcal{T}_{\sigma}$ is the Teichmüller space of the nodal surface $\Sigma_{\sigma}$. We also denote the set of all the elements $\sigma$ with $|\sigma|=1$ in $\mathcal{C}(\Sigma)$ by $\mathcal{S}$. Each element in $\mathcal{S}$ represent a single node. In [22], the author has shown that $\overline{\mathcal{T}}$ is a $\operatorname{CAT}(0)$ space, and each frontier stratum $\overline{\mathcal{T}}_{\sigma}$ is embedded totally geodesically into $\overline{\mathcal{T}}$, a generalization of a preceding result ([17]) by S. Wolpert which states that $\mathcal{T}$ is Weil-Petersson geodesically convex. In [23, a new space has been introduced which can be viewed as a Weil-Petersson geodesic completion, called Teichmüller-Coxeter complex $D(\overline{\mathcal{T}}, \iota)$. The space is a development of the original space $\overline{\mathcal{T}}$ by a Coxeter group generated by reflections across the frontier stratum $\left\{\overline{\mathcal{T}}_{\sigma}\right\}$. It was shown by Wolpert [19] that two intersecting strata of the same dimension meet at a right angle (in the sense of the Alexandrov angle between Weil-Petersson geodesics,) making the development $D(\overline{\mathcal{T}}, \iota)$ a so-called cubical complex [2, 5. This feature then is used to show that the development too is a CAT(0) space. The Coxeter complex setting allows to view the Teichmüller space as a convex set in an ambient space $D(\overline{\mathcal{T}}, \iota)$, bounded by a set of complex-codimension one "supporting hyperplanes" $\left\{D\left(\overline{\mathcal{T}}_{\sigma}, \iota\right)|| \sigma \mid=1\right\}$ of the frontier stratum $\left\{\overline{\mathcal{T}}_{\sigma}\right\}$ with each $\sigma$ representing a single node. Every boundary point is contained in at least one of the set of the supporting hyperplanes $\left\{D\left(\overline{\mathcal{T}}_{\sigma}, \iota\right)|| \sigma \mid=1\right\}$. In this picture, each $D\left(\overline{\mathcal{T}}_{\sigma}, \iota\right)$ is a totally geodesic set, metrically and geodesically complete, and when $D\left(\overline{\mathcal{T}}_{\sigma_{1}}, \iota\right)$ and $D\left(\overline{\mathcal{T}}_{\sigma_{2}}, \iota\right)$ intersect along $D\left(\overline{\mathcal{T}}_{\sigma_{1} \cup \sigma_{2}}, \iota\right)$, they meet at a right angle. One can view this as the translates of $\left\{D\left(\overline{\mathcal{T}}_{\sigma}, \iota\right)|| \sigma \mid=1\right\}$ by the action of the Coxeter group $W$ are forming a right-angled grid structure in $D(\overline{\mathcal{T}}, \iota)$, whose lattice points are the orbit image by the Coxeter group $W$ of the set $\left\{\overline{\mathcal{T}}_{\theta}|| \theta \mid=3 g-3\right\}$ with $\theta$ indexing the maximal set of nodes on the surface.

Under this setting, for each $\sigma$ with $|\sigma|=1$ one can consider a half space, namely the set $H_{\sigma}$, containing $\overline{\mathcal{T}}$ in the $D(\overline{\mathcal{T}}, \iota)$, and bounded by $D\left(\overline{\mathcal{T}}_{\sigma}, \iota\right)$. We note here the fact obtained by Wolpert [19] that the Weil-Petersson metric completion $\overline{\mathcal{T}}$ is the closure of the convex hull of the vertex set $\left\{\overline{\mathcal{T}}_{\theta}|| \theta \mid=\right.$ $3 g-3\}$, which suggests an interpretation of the Teichmüller space as a simplex.

We can summarize the discussion above as

$$
\overline{\mathcal{T}}=\cap_{\sigma \in \mathcal{S}} H_{\sigma} \quad \text { with } \quad \partial \overline{\mathcal{T}} \subset \cup_{\sigma} D\left(\overline{\mathcal{T}}_{\sigma}, \iota\right) .
$$

where every boundary point $b \in \partial \overline{\mathcal{T}}$ belongs to $D\left(\overline{\mathcal{T}}_{\sigma}, \iota\right)$ for some $\sigma$ in $\mathcal{S}$.

We now transcribe the Euclidean Funk geometry as well as its compatible Finsler structure in the previous section to the Weil-Petersson setting. There we demonstrated three different ways of writing down the Funk distance, which we called $F_{1}, F_{2}$ and $F_{3}$, all of which coincide. In the Weil-Petersson setting, they differ from each other, and they are related by inequalities.

\subsection{Weil-Petersson Funk metric $F_{2}$}

First note that as each $\overline{\mathcal{T}}_{\sigma}$ lies in $\overline{\mathcal{T}}$ as a complete convex set, for each point $x \in \overline{\mathcal{T}}$, there exists the nearest point projection $\Pi_{\sigma}(x) \in D\left(\overline{\mathcal{T}}_{\sigma}, \iota\right)$, and the 
Weil-Petersson geodesic $\overline{x \pi_{\sigma}(x)}$ meets with $D\left(\overline{\mathcal{T}}_{\sigma}, \iota\right)$ perpendicularly, its length uniquely realizing the distance $\inf _{y \in \overline{\mathcal{T}}_{\sigma}} d(x, y)=d\left(x, \Pi_{\sigma}(x)\right)$. We denote this number by $d\left(x, \overline{\mathcal{T}}_{\sigma}\right)$. We also introduce a notation $\nu_{\sigma}(x)$ for the unit vector at $x$ along the Weil-Petersson geodesic between $x$ and $\Pi_{\sigma}(x)$. In particular $-\nu_{\sigma}(x)$ is the Weil-Petersson gradient vector of the function $d\left(x, \overline{\mathcal{T}}_{\sigma}\right)$.

Also note that one can replace $\overline{\mathcal{T}}_{\sigma}$ by $D\left(\overline{\mathcal{T}}_{\sigma}, \iota\right)$ and get the same picture, for any $z \in \mathcal{T}$, we know that $\Pi_{\sigma}(z)$ is in $\mathcal{T}_{\sigma} \subset D\left(\iota, \overline{\mathcal{T}}_{\sigma}\right)$ due to the fact that the frontier sets intersect perpendicularly in terms of the Weil-Petersson Alexandorov angle. In other words, we have $\Pi_{\sigma}^{-1}\left[\mathcal{T}_{\sigma}\right]=\mathcal{T}$ for any $\sigma \in \mathcal{C}(\Sigma)$.

Definition We define the Weil-Petersson Funk metric $F_{2}$ on $\mathcal{T}$ as;

$$
F_{2}(x, y)=\sup _{\sigma \in \mathcal{S}} \log \frac{d\left(x, \overline{\mathcal{T}}_{\sigma}\right)}{d\left(y, \overline{\mathcal{T}}_{\sigma}\right)}
$$

In order to make the analogy with the Euclidean setting more obvious, and in order to make clearer the viewpoint that Teichmüller space is a convex body within an ambient space, we can instead define the metric, as

$$
F_{2}(x, y)=\sup _{\sigma \in \mathcal{S}} \log \frac{d\left(x, D\left(\overline{\mathcal{T}}_{\sigma}, \iota\right)\right)}{d\left(y, D\left(\overline{\mathcal{T}}_{\sigma}, \iota\right)\right)}
$$

The equality follows from the discussion in the paragraph preceding the definition of Weil-Petersson Funk metric.

Note that $F_{2}(x, y)$ satisfies the triangle inequality, for

$$
\begin{aligned}
F_{2}(x, y)+F_{2}(y, z) & =\sup _{\sigma \in \mathcal{S}} \log \frac{d\left(x, D\left(\overline{\mathcal{T}}_{\sigma}, \iota\right)\right)}{d\left(y, D\left(\overline{\mathcal{T}}_{\sigma}, \iota\right)\right)}+\sup _{\sigma \in \mathcal{S}} \log \frac{d\left(y, D\left(\overline{\mathcal{T}}_{\sigma}, \iota\right)\right)}{d\left(z, D\left(\overline{\mathcal{T}}_{\sigma}, \iota\right)\right)} \\
& \geq \sup _{\sigma \in \mathcal{S}}\left(\log \frac{d\left(x, D\left(\overline{\mathcal{T}}_{\sigma}, \iota\right)\right)}{d\left(y, D\left(\overline{\mathcal{T}}_{\sigma}, \iota\right)\right)}+\log \frac{d\left(y, D\left(\overline{\mathcal{T}}_{\sigma}, \iota\right)\right)}{d\left(z, D\left(\overline{\mathcal{T}}_{\sigma}, \iota\right)\right)}\right) \\
& =\sup _{\sigma \in \mathcal{S}} \log \frac{d\left(x, D\left(\overline{\mathcal{T}}_{\sigma}, \iota\right)\right)}{d\left(z, D\left(\overline{\mathcal{T}}_{\sigma}, \iota\right)\right)}=F_{2}(x, z)
\end{aligned}
$$

As in the Euclidean case, the equality holds when the stratum $D\left(\overline{\mathcal{T}}_{\sigma}, \iota\right)$ achieving the value of the supremum for $x$ and $y$ coincides with the one for $y$ and $z$. However, those strata are no longer characterized geometrically, which prevents us from stating that the Weil-Petersson geodesics are Funk distance minimizers. Recall in the Euclidean setting, the relevant supporting hypersurfaces are the ones containing the boundary points $b(x, y)$ and $b(y, z)$ where the rays hit the boundary set $\partial \Omega$.

We also remark that there is no definite statement for the convexity of the Funk distance $F_{2}(x, y)$ in the $y$ variable, unlike the Euclidean counterpart.

We estimate the Funk distance $F_{2}(x, y)$ from above and below by introducing two length metrics obtained by integrating linear structures $\tilde{p}_{\mathcal{T}, x}$ and $\hat{p}_{\mathcal{T}, x}$ corresponding to Funk metrics $F_{3}$ and $F_{1}$ in our Euclidean setting. 


\subsection{Finsler structure $\tilde{p}_{\mathcal{T}, x}$ and Funk metric $F_{3}$}

Definition Define the first Finsler structure by the following Minkowski function:

$$
\tilde{p}_{\mathcal{T}, x}(\xi)=\sup _{\sigma \in \mathcal{S}} \frac{\left\langle\nu_{\sigma}(x), \xi\right\rangle_{x}}{d\left(x, \overline{\mathcal{T}}_{\sigma}\right)}
$$

where the inner product is the Weil-Petersson pairing at $x$.

Note that the function $\tilde{p}_{\mathcal{T}, x}$ is convex in $\xi \in T_{x} \mathcal{T}$, for it is the supremum of linear (in particular convex) functions. Let $\alpha:[0,1] \rightarrow \mathcal{T}$ be a $C^{1}$-path connecting $x=\alpha(0)$ and $y=\alpha(1)$. Then the length of $\alpha$ according to the Minkowski function $\tilde{p}_{\mathcal{T}, x}$ is defined as

$$
\tilde{\mathrm{L}}(\alpha)=\int_{0}^{1} \tilde{p}_{\mathcal{T}, \alpha(t)}(\dot{\alpha}(t)) d t
$$

This in turn defines a distance function

$$
F_{3}(x, y)=\inf _{\alpha} \tilde{L}(\alpha)
$$

with $\alpha(0)=x, \alpha(1)=y$.

We show that the Funk distance $F_{2}(x, y)$ can be estimated from above by the length $\tilde{L}(\alpha)$;

$$
\begin{aligned}
F_{2}(x, y) & =\sup _{\sigma \in \mathcal{S}} \log \frac{d\left(\alpha(0), \mathcal{T}_{\sigma}\right)}{d\left(\alpha(1), \mathcal{T}_{\sigma}\right)} \\
& =\sup _{\sigma \in \mathcal{S}}\left[-\left.\log d\left(\alpha(t), \mathcal{T}_{\sigma}\right)\right|_{0} ^{1}\right]=\sup _{\sigma \in \mathcal{S}} \int_{0}^{1} \frac{\left\langle\nu_{\sigma}(\alpha(t)), \dot{\alpha}(t)\right\rangle_{\alpha(t)}}{d\left(\alpha(t), \overline{\mathcal{T}}_{\sigma}\right)} d t \\
& \leq \int_{0}^{1} \sup _{\sigma \in \mathcal{S}} \frac{\left\langle\nu_{\sigma}(\alpha(t)), \dot{\alpha}(t)\right\rangle_{\alpha(t)}}{d\left(\alpha(t), \overline{\mathcal{T}}_{\sigma}\right)} d t=\int_{0}^{1} \tilde{p}_{\mathcal{T}, \alpha(t)}(\dot{\alpha}(t)) d t=\tilde{\mathrm{L}}(\alpha)
\end{aligned}
$$

By taking infimum over the piecewise $C^{1}$ paths $\alpha$ 's of the inequality, we obtain the estimate

$$
F_{2}(x, y) \leq F_{3}(x, y)
$$

\subsection{Linear structure $\hat{p}_{\mathcal{T}, x}$ and length metric $F_{1}$}

Definition For a pair of distinct points $x$ and $y$ in $\mathcal{T}$, with the ray from $x$ through $y$ hitting a strata $\mathcal{T}_{\sigma_{x y}}$, we define a number $\phi_{1}(x, y)$ by

$$
\phi_{1}(x, y)=\log \frac{d\left(x, T\left(x, \xi_{x y}, \sigma_{x y}\right)\right)}{d\left(y, T\left(x, \xi_{x y}, \sigma_{x y}\right)\right)}
$$

where $T(x, \xi, \sigma)$ is the point(s) where the ray $\left\{\exp _{x} t \xi \mid t \geq 0\right\}$ and $D\left(\overline{\mathcal{T}}_{\sigma}, \iota\right)$ meet and $\xi_{x y}$ is the tangent vector in $T_{x} \mathcal{T}$ such that $\exp _{x} \xi_{x y}=y$. If the ray $\left\{\exp _{x} t \xi \mid t \geq 0\right\}$ does not hit any strata, then we set $\phi_{1}(x, y)=0$ 
The ray staying in the interior $\mathcal{T}$ can indeed occur, when for example $x$ and $y$ are on a pseudo-Anosov axis [6, 19] for some pseudo-Anosov element $\gamma$ in the mapping class group of $\Sigma$. It is not known that $\phi_{1}(x, y)$ satisfies the triangle inequality satisfied by its Euclidean analogue $F_{1}(x, y)$.

Definition We now define another auxiliary linear structure on each tangent space;

$$
\hat{p}_{\mathcal{T}, x}(\xi)=\sup _{\sigma \in \mathcal{S}} \frac{\|\xi\|}{d(x, T(x, \xi, \sigma))}
$$

where $\|\xi\|$ is the Weil-Petersson norm of $\xi$. When the ray $t \xi$ hits no strata, set $\hat{p}_{\mathcal{T}, x}(\xi)=0$

The functional $\hat{p}_{\mathcal{T}, x}$ is neither continuous nor convex on $T_{x} \mathcal{T}$, hence does not induce a Finsler structure. Once the geodesic ray in $D(\overline{\mathcal{T}}, \iota)$ crosses the bordification set $\partial \mathcal{T} \overline{\mathcal{T}} \mathcal{T}$, the exponential map $\exp _{x}: T_{x} \mathcal{T} \rightarrow D(\overline{\mathcal{T}}, \iota)$ is no longer single valued. Hence for a given $\sigma, T(x, \xi, \sigma)$ is possibly a set of points. However, in $\overline{\mathcal{T}}$ the exponential map is single-valued. Hence the value of $\sup _{\sigma \in \mathcal{S}} 1 / d(x, T(x, \xi, \sigma))$ is the reciprocal of the Weil-Petersson distance between $x$ and the bordification set $\partial \mathcal{T}$ along the ray $\left\{\exp _{x} t \xi \mid t>0\right\}$. Here we note when the ray from $x$ through $y$ hits the strata $\mathcal{T}_{\sigma_{x y}}$, the integral of the new linear structure along the Weil-Petersson geodesic $\exp _{x} t \xi_{x y}$, which we denote $s(t)$ below, coincides with the number $\phi_{1}(x, y)$;

$$
\phi_{1}(x, y)=\int_{0}^{1} \hat{p}_{\overline{\mathcal{T}}, s(t)}(\dot{s}(t)) d t
$$

with $s(1)=y$, as the $t$-derivative of $\phi_{1}(x, s(t))$ is equal to $\hat{p}_{\mathcal{T}, s(t)}(\dot{s}(t))$

This linear structure $\hat{p}_{\mathcal{T}, x}$ defines a length for each piecewise $C^{1}$-path connecting $x=\alpha(0)$ and $y=\alpha(1)$;

$$
\hat{L}(\alpha)=\int_{0}^{1} \hat{p}_{\mathcal{T}, \alpha(t)}(\dot{\alpha}(t)) d t
$$

Then we have another weak metric $F_{1}(x, y)$ by

$$
F_{1}(x, y)=\inf _{\alpha} \hat{L}(\alpha)
$$

where the infimum is taken over the set of piecewise $C^{1}$-paths connecting $x=$ $\alpha(0)$ and $y=\alpha(1)$. That this is a weak metric is clear as the two conditions 1 and 2 in the definition of metric are trivially met. When the geodesic ray from $x$ through $y$ stay in $\mathcal{T}$ indefinitely, $F_{1}(x, y)=0$ as the ray has zero length with respect to the length defined by $\hat{p}_{\mathcal{T}, x}$.

We show that the Funk distance $F_{2}(x, y)$ can be estimated from below by $F_{1}(x, y)$. As before, let $s(t)=\exp _{x} t \xi_{x y}$, and assume it hits $\mathcal{T}_{\sigma_{x y}}$, for otherwise 
$F_{1}(x, y)=0$ and the comparison is trivial.

$$
\begin{aligned}
F_{2}(x, y) & =\sup _{\sigma \in \mathcal{S}} \log \frac{d\left(\alpha(0), \mathcal{T}_{\sigma}\right)}{d\left(\alpha(1), \mathcal{T}_{\sigma}\right)} \geq \log \frac{d\left(\alpha(0), \mathcal{T}_{\sigma_{x y}}\right)}{d\left(\alpha(1), \mathcal{T}_{\sigma_{x y}}\right)}=\log \frac{d\left(x, \mathcal{T}_{\sigma_{x y}}\right)}{d\left(y, \mathcal{T}_{\sigma_{x y}}\right)} \\
& \geq \log \frac{d\left(x, T\left(x, \xi, \sigma_{x y}\right)\right)}{d\left(y, T\left(x, \xi, \sigma_{x y}\right)\right)}=\phi_{1}(x, y)=\int_{0}^{1} \frac{\|\dot{s}(t)\|}{d\left(s(t), T\left(x, \xi, \sigma_{x y}\right)\right)} d t \\
& =\int_{0}^{1} \hat{p}_{\mathcal{T}, s(t)}(\dot{s}(t)) d t=\hat{L}(s) \geq \inf _{\alpha} \hat{L}(\alpha) \\
& =F_{1}(x, y)
\end{aligned}
$$

The second inequality follows from the following argument. First the WeilPetersson distance $d\left(\alpha(t), \mathcal{T}_{\sigma}\right)$ is strictly convex in $t$, which says that

$$
\left\langle\nabla_{\dot{\alpha}(t)} \nu_{\sigma}(\alpha(t)), \dot{\alpha}(t)\right\rangle>0
$$

which implies that the angle between $\nu_{\sigma}$ and $\dot{\alpha}(t)$ is increasing in $t$, as the quantity $\left\langle\nu_{\sigma}(\alpha(t)), \dot{\alpha}(t)\right\rangle$ is proportional to the cosine of the angle. Consider two right triangles the former being the geodesic triangle in $\overline{\mathcal{T}}$ whose vertices are $x, T\left(x, \xi, \sigma_{x y}\right)$ and $\Pi_{\sigma_{x y}}(x)$ (the foot of $\left.x\right)$, and the latter being the Euclidean right triangle with a side of length $d\left(x, \Pi_{\sigma_{x y}}(x)\right)$ between the vertex of a right angle and a vertex of an angle equal to the Weil-Petersson angle at $x$ of the former geodesic triangle in $\overline{\mathcal{T}}$.

The monotone increasing property of the angle between $\nu_{\sigma}(\alpha(t))$ and $\dot{\alpha}(t)$ contrasted to the constancy of the corresponding angles in the comparison triangle in $\mathbf{R}^{2}$, one concludes that

$$
\frac{d\left(x, \mathcal{T}_{\sigma_{x y}}\right)}{d\left(y, \mathcal{T}_{\sigma_{x y}}\right)}>\frac{d\left(x, T\left(x, \xi, \sigma_{x y}\right)\right)}{d\left(y, T\left(x, \xi, \sigma_{x y}\right)\right)} .
$$

This completes the proof of the inequality $F_{2} \geq F_{1}$.

Hence we summarize the Weil-Petersson situation as follows.

Theorem 2 On the Teichmüller space $\mathcal{T}$, the Funk metric $F_{2}$ and the Funk metrics $F_{1}$ and $F_{3}$ induced by $\hat{p}_{\mathcal{T}, x}$ and $\tilde{p}_{\mathcal{T}, x}$ respectively as above have the following comparison;

$$
F_{1}(x, y) \leq F_{2}(x, y) \leq F_{3}(x, y) .
$$

Furthermore, each metric is invariant under the extended mapping class group action.

We recall that the extended mapping class group is the full Weil-Petersson isometry group of $\mathcal{T}(12]$.)

\subsection{Weil-Petersson Hilbert metric on $\mathcal{T}$}

We symmetrize the Weil-Petersson Funk metric $F_{2}$ by taking the arithmetic means. 
Definition We set

$$
H(x, y)=\frac{1}{2}\left(F_{2}(x, y)+F_{2}(y, x)\right)
$$

and call this Weil-Petersson Hilbert metric on the Teichmüller space $\mathcal{T}$.

This metric satisfies the triangle inequality. It is obtained by taking the sum of the following pair of inequalities $F_{2}(x, y)+F_{2}(y, z) \geq F_{2}(x, z)$ which was shown above, and $F_{2}(z, y)+F_{2}(y, x) \geq F_{2}(z, x)$ which follows from the same argument as in the proof of the triangle inequality in the Euclidean setting.

This provides a solution to a problem raised by A.Papadopoulos [13] "Realize Teichmüller space as a bounded convex set somewhere and study the Hilbert metric on it" as in our study, the Teichmüller space is realized as a convex set, however unbounded, in the Teichmüller-Coxeter space, on which we have defined a Hilbert metric.

We briefly remark that there are other ways of symmetrize the Funk metric, one of which is to define a new metric by $D(x, y)=\max \{F(x, y), F(y, x)\}$.

\subsection{Concluding remarks}

On the closed surface $\Sigma$ as above, consider a pair of points in the Teichmüller space $\mathcal{T}$, represented by hyperbolic metrics $x$ and $y$. Then Thurston's asymmetric metric [16] is defined by

$$
K(x, y)=\sup _{\sigma \in \mathcal{S}} \log \frac{\ell_{x}(\sigma)}{\ell_{y}(\sigma)}
$$

where $\ell_{x}(\sigma)$ denotes the hyperbolic length of the simple closed geodesic $\sigma$ on the hyperbolic surface $(\Sigma, x)$. The Finsler structure of this metric is described on p.20 of [16]; for tangent vector $Y$ the Finsler norm is given by $\|Y\|=\sup _{\lambda}\left(\frac{Y(\text { length }(\lambda)}{\operatorname{length}(\lambda)}\right)$ where $\lambda$ is taken over the space of measured geodesic laminations, corresponding to our Weil-Petersson Finsler structure $\tilde{p}_{\mathcal{T}, x}(\xi)=$ $\sup _{\sigma} \frac{\left\langle\nu_{\sigma}(x), \xi\right\rangle_{x}}{d\left(x, \overline{\mathcal{T}}_{\sigma}\right)}$. Note also that $\left(\frac{Y(\operatorname{length}(\lambda)}{\operatorname{length}(\lambda)}\right)(x)$ is the directional derivative of $\log \frac{\operatorname{length}(\lambda)(y)}{\operatorname{length}(\lambda)(x)}$ by $Y$ at $x$, while $\frac{\left\langle\nu_{\sigma}(x), \xi\right\rangle_{x}}{d\left(x, \overline{\mathcal{T}}_{\sigma}\right)}$ is the directional derivative of $\log \frac{d\left(y, \overline{\mathcal{T}}_{\sigma}\right)}{d\left(x, \overline{\mathcal{T}}_{\sigma}\right)}$ by $\xi$ at $x$.

The asymptotic expansion (22]) of the Weil-Petersson metric near a strata $\mathcal{T}_{\sigma}$ states that the Weil-Petersson metric tensor has an almost-product structure there, of the Weil-Petersson metric of the frontier strata $\mathcal{T}_{\sigma}$ and the part expressed by the Fenchel-Nielsen parameters $\left(\ell_{\sigma}, \theta_{\sigma}\right)$. The second part is of the form

$$
4 \pi^{3}\left(d \rho_{\sigma}^{2}+\frac{1}{4} \rho_{\sigma}^{6} d \theta_{\sigma}^{2}\right)
$$

where $\rho_{\sigma}=\sqrt{\ell_{\sigma}}$. The constant $4 \pi^{3}$ is due to S.Wolpert([19.) This in turn says that near each stratum, the Weil-Petersson distance $d\left(x, \mathcal{T}_{\sigma}\right)$ is approximated 
by $\sqrt{\ell_{\sigma}(x)}$. This observation can be transcribed to the following comparison;

$$
\log \frac{d\left(x, \overline{\mathcal{T}}_{\sigma}\right)}{d\left(y, \overline{\mathcal{T}}_{\sigma}\right)} \approx \log \frac{\sqrt{\ell_{x}(\sigma)}}{\sqrt{\ell_{y}(\sigma)}}=\frac{1}{2} \log \frac{\ell_{x}(\sigma)}{\ell_{y}(\sigma)}
$$

provided that $x$ and $y$ are sufficiently close to $\overline{\mathcal{T}}_{\sigma}$ for some $\sigma$, an observation associating the two metrics $F_{2}$ and $K$.

Most likely it is too optimistic to hope for the two metric $F_{2}$ and $K$ to coincide with each other (up to the constant $1 / 2$.) However, this leads to a speculation as to whether the ratio $d\left(x, \overline{\mathcal{T}}_{\sigma}\right) / d\left(y, \overline{\mathcal{T}}_{\sigma}\right)$ can be seen as a number encoding some information about an appropriate geodesic lamination relevant to the pair of hyperbolic metrics $x$ and $y$ as well as the class $\sigma$ in $\mathcal{S}$.

Lastly, in addition to the similarity to Thurston's metric, the form of the Weil-Petersson Funk metric $F_{2}$ should be contrasted with Teichmüller metric defined on Teichmüller spaces, in a particular representation found by Kerckhoff [9];

$$
d_{T}\left(\left[G_{1}\right],\left[G_{2}\right]\right)=\frac{1}{2} \sup _{\sigma \in \mathcal{S}} \log \frac{\operatorname{Ext}_{\left[G_{1}\right]}(\sigma)}{\operatorname{Ext}_{\left[G_{2}\right]}(\sigma)}
$$

where $\operatorname{Ext}_{[G]}(\sigma)$ is the extremal length of $\sigma$ with respect to the conformal structure $[G]$.

\section{References}

[1] W. Abikoff, Degenerating families of Riemann surfaces. Ann. of Math. 105 (1977), 29-44.

[2] M. Bridson and A. Haefliger. Metric spaces of non-positive curvature. Springer, Berlin (1999).

[3] H. Busemann and W. Mayer. On the foundations of calculus of variations. Trans. Amer. Math. Soc. 49, (1941) 173-198.

[4] A. Belkhirat, A. Papadopoulos and M. Troyanov. Thurston's weak metric on the Teichmüller space of the torus. Trans. Amer. Math. Soc. 357 (2005), no. 8, 3311-3324.

[5] M. Davis, M. The geometry and topology of Coxeter groups. Princeton University Press, Princeton (2007).

[6] G. Daskalopoulos and R. Wentworth. Classification of Weil-Petersson isometries. Amer. J. Math. 125 (2003), 941-975.

[7] P.Funk. Über geometrien, bei denen die geraden die kürzesten sind. Math. Ann. 101 (1929), 226-237.

[8] D.Hilbert. Über die gerade Linie als kürzeste Verbindung zweier Punkte. Math. Ann. 46 (1895) 91-96. 
[9] S. Kerckhoff, The asymptotic geometry of Teichmüller space. Ann. of Math. 66 (1980), 235-265.

[10] P. Kelly and E. Straus. Curvature in Hilbert geometries. Pacific J. Math. 8 (1958), 119-125.

[11] H. Masur, The extension of the Weil-Petersson metric to the boundary of the Teichmüller space. Duke Math. J. 43 (1976), 623-635.

[12] H.Masur and M.Wolf. The Weil-Petersson isometry group. Geom.Dedicata, 93(2002), 177-190.

[13] A. Papadopoulos. Problem 13 in Problem Session "Teichmüller Theory", Oberwolfach Reports Vol. 7, (2010) Issue 4, 3085-3157.

[14] A. Papadopoulos and M. Troyanov. Weak Finsler structures and the Funk weak metric. Math. Proc. Cambridge Philos. Soc. 147 (2009), no. $2,419-437$.

[15] A. Papadopoulos and M. Troyanov. Harmonic symmetrization of convex sets and of Finsler structures, with applications to Hilbert geometry. Expo. Math. 27 (2009), no. 2, 109-124.

[16] W. Thurston. Minimal stretch maps between hyperbolic surfaces. Preprint, (1986), available at arxiv:math GT/9801039.

[17] S. Wolpert. Geodesic Length Functions and the Nielsen Problem. J. Diff. Geom. 25 (1987), 275-295.

[18] S. Wolpert. Geometry of the Weil-Petersson completion of Teichmüller space. In: Surveys in differential geometry VIII: papers in honor of Calabi, Lawson, Siu and Uhlenbeck. Intl. Press, Cambridge, MA (2003).

[19] S. Wolpert. Behavior of geodesic-length functions on Teichmüller space. J. Diff. Geom. 79, (2008) 277-334.

[20] S. Wolpert. Families of Riemann surfaces and Weil-Petersson geometry. CBMS Regional Conference Series in Mathematics, 113. American Mathematical Society, Providence, RI, (2010).

[21] S. Yamada. Weil-Petersson convexity of the energy functional on classical and universal Teichmüller spaces. J. Differential Geom. 51 (1999) $35-96$.

[22] S.Yamada. On the geometry of Weil-Petersson completion of Teichmüller spaces. Math. Res. Lett. 11 (2004), 327-344.

[23] S.Yamada. Weil-Petersson geometry of Teichmüller-Coxeter complex and its finite rank property. Geom. Dedicata 145 (2010), 43-63. 